\title{
Morphology of a highly asymmetric double crystallizable poly ( $\varepsilon$-caprolactone-b-ethylene oxide) block copolymer
}

\author{
Liangbin $\mathrm{Li}^{\mathrm{a})}$ \\ National Synchrotron Radiation Laboratory, University of Science and Technology of China, Anhui 230026, \\ China and Department of Polymer Science and Engineering, University of Science and Technology \\ of China, Anhui 230026, China
}

Fenghua Meng and Zhiyuan Zhong

Department of Polymer Chemistry and Biomaterials, Faculty of Science and Technology, University of Twente, 7500 AE Enschede, The Netherlands and Institute for Biomedical Technology, Faculty of Science and Technology, University of Twente, 7500 AE Enschede, The Netherlands

Dmytro Byelov and Wim H. de Jeu

FOM-Institute for Atomic and Molecular Physics, Kruislaan 407, 1098 SJ Amsterdam, The Netherlands

\section{Jan Feijen}

Department of Polymer Chemistry and Biomaterials, Faculty of Science and Technology, University of Twente, 7500 AE Enschede, The Netherlands and Institute for Biomedical Technology, Faculty of Science and Technology, University of Twente, 7500 AE Enschede, The Netherlands

(Received 26 September 2006; accepted 13 November 2006; published online 11 January 2007)

The morphology of a highly asymmetric double crystallizable poly( $\varepsilon$-caprolactone- $b$-ethylene oxide) (PCL- $b$-PEO) block copolymer has been studied with in situ simultaneously small and wide-angle x-ray scattering as well as atomic force microscopy. The molecular masses $M_{n}$ of the PCL and PEO blocks are 24000 and 5800, respectively. X-ray scattering and rheological measurements indicate that no microphase separation occurs in the melt. Decreasing the temperature simultaneously triggers off a crystallization of PCL and microphase separation between the PCL and PEO blocks. Coupling and competition between microphase separation and crystallization results in a morphology of PEO spheres surrounded by PCL partially crystallized in lamella. Further decreasing temperature induces the crystallization of PEO spheres, which have a preferred orientation due to the confinements from hard PCL crystalline lamella and from soft amorphous PCL segments in different sides. The final morphology of this highly asymmetric block copolymer is similar to the granular morphology reported for syndiotactic polypropylene and other (co-) polymers. This implies a similar underlying mechanism of coupling and competition of various phase transitions, which is worth further exploration. (C) 2007 American Institute of Physics. [DOI: 10.1063/1.2405357]

\section{INTRODUCTION}

Coupling and competition between different phase transitions has been widely applied to create ordered structures over various length scales and may hold the key to the development of new structures needed for advanced devices. ${ }^{1,2}$ In this context, the richness of phase transitions in block copolymers provides an inexhaustible pool for materials science. The crystallization behavior of block copolymers with one crystallizable block has been extensively studied, especially regarding the effect of the confinement of the crystallization by the amorphous block. ${ }^{3-9}$ Only a few studies provide a more generic idea concerning dynamic coupling between crystallization and microphase separation. ${ }^{10,11}$ More recently, block copolymers with multiple crystallizable blocks have attracted attention. ${ }^{12-24}$ Such systems may not only provide new insight on polymer crystallization but also lead to new possibilities to tune microstructures on the

\footnotetext{
${ }^{a)}$ Author to whom correspondence should be addressed. Electronic mail: lbli@ustc.edu.cn
}

nanometer scale. However, so far most emphasis has been on the crystallization kinetics rather than on the final morphology, though the latter is more important for nanostructural design.

Poly( $\varepsilon$-caprolactone) (PCL) and poly(ethylene oxide) (PEO) are crystallizable polymers that are known to be biocompatible. ${ }^{25,26}$ Copolymers consisting of both PCL and PEO segments (blocks) exhibit drug permeability and degradability and have been proposed for a wide range of medical applications. Asymmetric PCL- $b$-PEO block copolymer can form polymersome in water, ${ }^{27}$ which is expected to be a good drug carrier. Both drug permeability and biodegradability depend on the crystallinity. ${ }^{26}$ Nevertheless, only a few studies have been published on the morphology and the crystallization (melting) properties of such block copolymers. $^{28-30}$ The early example by Perret and Skoulios ${ }^{28}$ gives detailed information about the crystallization and the lamellae structure of PCL-PEO-PCL triblock copolymers for PEO with a molecular weight $M_{w}=8600$ and different lengths of the PCL blocks. The crystallization properties of 
TABLE I. The physical parameters of the block copolymer material. (The volume fraction and molecular weight are from NMR. Amorphous and crystalline densities of PCL are from Refs. 47 and 48 while amorphous and crystalline densities of PEO are from Ref. 49. The equilibrium melting point is from Ref. 50 for PCL and from Ref. 51 for PEO as a homopolymer with similar molecular weight.)

\begin{tabular}{lll}
\hline \hline & PEO & PCL \\
\hline Volume fraction & $17 \%$ & $83 \%$ \\
Molecular weight $M_{n}$ & 5800 & 24000 \\
Amorphous density $\rho_{a}$ at $60{ }^{\circ} \mathrm{C}(\mathrm{g} / \mathrm{ml})$ & 1.092 & 1.11 \\
Crystalline density $\rho_{c}(\mathrm{~g} / \mathrm{ml})$ & 1.203 & 1.201 \\
Equilibrium melting temperature $T_{m}^{0}\left({ }^{\circ} \mathrm{C}\right)$ & 64 & 77 \\
\hline \hline
\end{tabular}

this system were reported to depend on the length of each block and the crystallization temperature. ${ }^{27-30}$ Gan et al. ${ }^{31}$ studied the isothermal crystallization and melting behavior of PCL- $b$-PEO diblock copolymers. The PCL block was crystallizable for a weight fraction of the PEO block below $20 \%$. The crystallization kinetics of highly asymmetric block copolymers PCL- $b$-PEO with different configurations was studied by Bogdanov et al. ${ }^{16}$ The authors proposed a superlattice lamellar morphology of crystallized PCL and PEO layers. Though it is rather natural to expect polymer crystallization to result in a lamellar morphology, no direct experimental evidence was given to justify the particular picture in this case.

Asymmetric block copolymers provide model systems for phase transitions in common crystalline polymers. Though common polymers such as isotactic polypropylene (iPP) and high-density polyethylene (HDPE) are recognized as homopolymers, many defects such as tacticity, comonomers, branches, and loops exist in the molecular chains. For example, most commercial iPP has an isotacticity around 95\%-98\%. Hence iPP is actually a highly asymmetric multiblock copolymer containing blocks (segments) with and without chain defects as determined by the tacticity distribution. Since such a common "homopolymer" has a wide distribution of block length, any effect such as phase separation is easily blurred by crystallization. Such hidden phenomena may be revealed by a study on the highly asymmetric block copolymers with narrow block length distribution, which have a sharper phase transition temperature and a regular morphology representing the underlying physical mechanisms. $^{3}$

In this work, the crystallization behavior and morphology of PCL- $b$-PEO block copolymers with molecular weights $M_{n, \mathrm{PCL}}=24000$ and $M_{n, \mathrm{PEO}}=5800$ (see Table I) were studied with small- and wide-angle X-ray scattering (SAXS-WAXS) as well as atomic force microscopy (AFM). Experimental results show that after crystallization of PCL a superlattice is formed by PCL lamellae and PEO spheres. This picture does not follow simple expectations of crystallization alone, but can be understood rather nicely from coupling and competition between crystallization and microphase separation.

\section{EXPERIMENTAL SECTION}

\section{Materials}

The block copolymer PCL- $b$-PEO was synthesized, as reported previously, ${ }^{27}$ by ring-opening polymerization of $\varepsilon$-caprolactone (CL) using zinc bis[bis(trimethysilyl)amide] and monomethoxy PEO as an initiator. Polymerization was conducted at room temperature using dichloromethane as a solvent. $\varepsilon$-caprolactone (Merck) and dichloromethane (Biosolve) were dried over $\mathrm{CaH}_{2}$ and distilled prior to use. Monomethoxy PEO with $M_{n}=5800$ (Fluka) was dried by dissolution in anhydrous toluene followed by azeotropic distillation under $\mathrm{N}_{2}$; bis[bis(trimethysilyl)amide] (Aldrich) was used as received. A complete monomer conversion was obtained within $2 \mathrm{~h}$. NMR and gel permeation chromatography (GPC) measurements showed that the PCL- $b$-PEO block copolymer had $M_{n}=29800$ and a polydispersity of 1.9 .

\section{Characterization}

Simultaneous WAXS and SAXS measurements were made using an in-house setup at the FOM-Institute for Atomic and Molecular Physics (Amsterdam) with a rotating anode X-ray generator (Rigaku RU-H300, 18 kW) equipped with two parabolic multilayer mirrors (Bruker, Karlsruhe), giving a highly parallel beam (divergence of about $0.02^{\circ}$ ) of monochromatic $\mathrm{Cu} K_{\alpha}$ radiation $(\lambda=0.154 \mathrm{~nm})$. The SAXS intensity was collected with a two-dimensional gas-filled wire detector (Bruker Hi-Star). A semitransparent beamstop placed in front of the area detector allowed monitoring the intensity of the direct beam. The WAXS intensity was recorded with a linear position sensitive detector $(50 \mathrm{M}, \mathrm{M}$. Braun, Germany), which could be rotated around the beam path to measure either in the meridianal or in the equatorial direction. The SAXS and WAXS intensities were normalized to the intensity of the direct beam.

\section{Experimental procedure}

A Linkam CSS450 system was employed as a temperature-controlled sample stage. Samples were kept in a brass sample holder with kapton windows replacing the original glass windows of the system. For isothermal crystallization the sample was first heated up to $80{ }^{\circ} \mathrm{C}$ for $10 \mathrm{~min}$ (above the nominal melting point of both blocks, see Table I) and subsequently cooled down to the crystallization temperature. A nitrogen atmosphere was used to prevent possible degradation at high temperatures. SAXS and WAXS measurements were taken during isothermal crystallization with 30 and $120 \mathrm{~s} /$ frame, respectively. We let the PCL blocks crystallize first, typically at temperatures around $40-50{ }^{\circ} \mathrm{C}$. After completion of the crystallization of PCL as indicated by saturation of the SAXS intensity, the sample was cooled down to a lower temperature where the PEO block could crystallize (typically below room temperature). Any transitions during heating were also studied by x-ray scattering. 

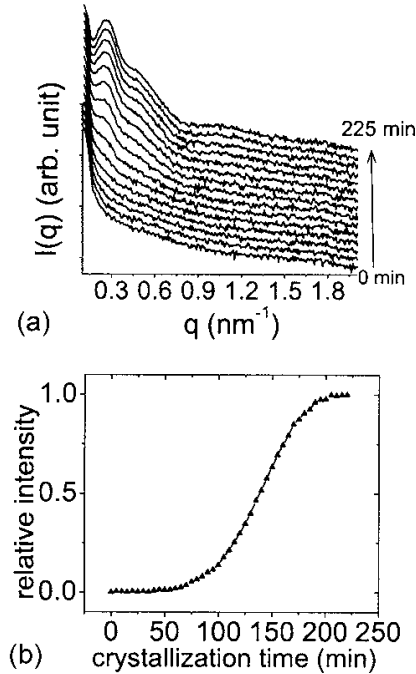

FIG. 1. (a) SAXS patterns of PCL- $b$-PEO during isothermal crystallization at $55^{\circ} \mathrm{C}$. (b) Relative SAXS intensity vs crystallization time.

\section{Atomic force microscopy}

AFM measurements were carried out with a Solver Scanning Force Apparatus (SFA) (NT-MDT, Zelenograd, Moscow) in the tapping mode using a scanning cantilever. In this mode the AFM contrast of the phase image is due to different mechanical properties of the crystalline and the amorphous regions. Fresh surfaces representing the bulk morphology were obtained by cutting the crystallized samples using a homemade cryogenic microtome setup.

\section{RESULTS}

The SAXS patterns of the PEO- $b$-PCL during isothermal crystallization at $55^{\circ} \mathrm{C}$ are shown in Fig. 1(a). Before the onset of crystallization, the SAXS intensity pattern shows no scattering peak. Though it suggests a homogeneous phase before crystallization, we cannot be sure because the density contrast between amorphous PCL and PEO is small (see Table I). Hence a possible phase separation might not be visible in SAXS. However, the initial mixed state of the PEO- $b$-PCL block copolymer is confirmed by the rheological measurements of Fig. 2 that show no transition before the onset of crystallization. Thus the crystallization does take place in a homogeneous phase rather than in the microphase separated domains. As PCL is the majority block and occupies more than $80 \%$ of volume, we anticipate the crystallized

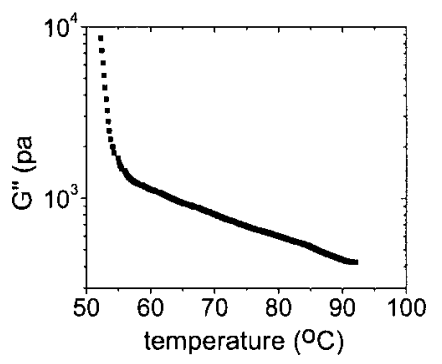

FIG. 2. Loss modulus $\mathrm{G}^{\prime \prime}$ of PCL- $b$-PEO during slow cooling with a rate of $0.5^{\circ} \mathrm{C} / \mathrm{min}$.
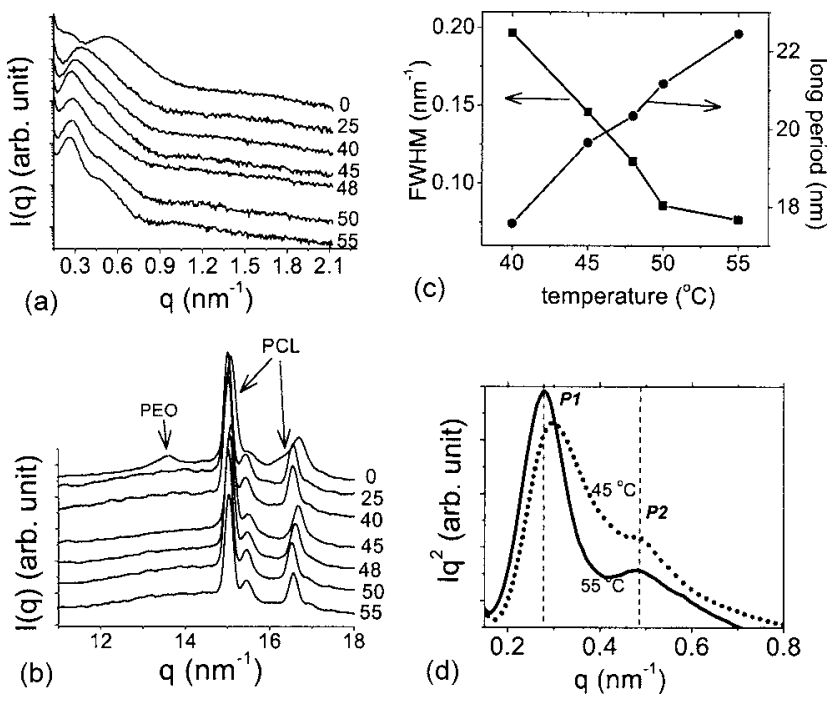

FIG. 3. (a) SAXS and (b) WAXS patterns of PCL- $b$-PEO crystallized at different temperatures. (c) Peak width and long period as obtained from the first SAXS peak of the samples used. (d) Lorentzian corrected $\left(I q^{2}\right)$ SAXS patterns of the samples crystallized at 45 and $55^{\circ} \mathrm{C}$.

block to be PCL and not PEO, whose assignment is supported by the WAXS measurements of Fig. 3(b).

The variation in the scattered SAXS intensity with crystallization time [Fig. 1(b)] provides information on the kinetics of crystallization. The growth kinetics has been analyzed with the Avrami equation $x_{c}=1-\exp \left(-k t^{n}\right)$, where $x_{c}$ is the crystallinity, $t$ the crystallization time, $k$ the rate constant, and $n$ the so-called Avrami index. A linear fit of $\ln \left(-\ln \left(1-x_{c}\right)\right)$ vs $\ln t$ gives $n=4$. The temperature dependence of the long spacing (P1) and of the Avrami coefficient $(n=4)$ perfectly agree with previous measurements on pure PCL homopolymer and indicate that the PEO block does not impose geometric constraints. ${ }^{32,33}$ The crystallization kinetics of double crystallizable block polymers having been studied by several groups before, ${ }^{13-24}$ we shall now focus on the morphology development.

Figures 3(a) and 3(b) display the final SAXS and WAXS patterns after crystallizing at different temperatures as indicated. Below $40^{\circ} \mathrm{C}$, it was not possible to conduct an isothermal crystallization process anymore due to the limited cooling rate of the sample stage. The results at $0{ }^{\circ} \mathrm{C}$ were obtained by quenching in liquid $\mathrm{N}_{2}$ and by subsequent annealing at $0{ }^{\circ} \mathrm{C}$. The WAXS measurements down to room temperature reveal that only the PCL block crystallizes and the PEO block is still amorphous. Only for the sample annealed at $0{ }^{\circ} \mathrm{C}$ both blocks have crystallized.

Within the experimentally isothermal crystallization temperature range $\left(40-55^{\circ} \mathrm{C}\right)$, the $q$ value and the width of the first SAXS peak (P1) decrease with increasing crystallization temperature [see Fig. 3(c)]. The long spacing varies effectively linearly with the crystallization temperature, following the same general rule as homopolymer crystallization. Again the presence of the PEO block does not seem to have any effect on the crystallization behavior of the PCL block, in spite of the covalent bonding connecting the two blocks. Nevertheless, the increase of the long spacing due to more favorable thicker PCL crystals should stretch the PEO 

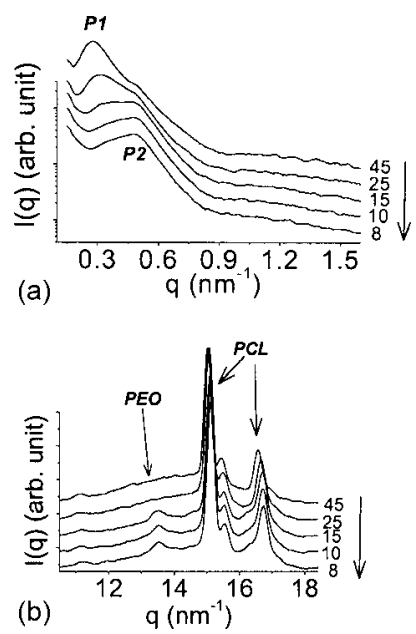

FIG. 4. (a) SAXS and (b) WAXS patterns during stepwise cooling of PCL$b$-PEO initially crystallized at $45{ }^{\circ} \mathrm{C}$.

chains. The resulting deviations from a Gaussian configuration necessarily lead to an entropic penalty. ${ }^{34-37}$ Evidently this entropic penalty must be small compared to the enthalpy gain, as no effect on the long spacing is observable.

Though there is little doubt about the lamellar PCL crystals, the overall morphology of the PEO- $b$-PCL block copolymer is not obvious. Following the homopolymer type of crystallization kinetics and the temperature dependence of long spacing, there is a tendency to assume that the crystallized morphology is overall lamellar, as other authors suggested. ${ }^{16}$ However, at $17 \%$ volume fraction of PEO the microphase-separated morphology should be located in the sphere region of the generic phase diagram of block copolymers. ${ }^{3}$ A closer scrutiny of the SAXS curves of Figs. 1(a) and 3(a) indicates, apart from the main peak, a second shoulder at larger $q$ values. If it is just the simple lamellar structure, the Lorentzian correction should be applied to obtain the structure factor. Figure 3(d) shows SAXS curves from samples crystallized at 55 and $45^{\circ} \mathrm{C}$ after a Lorentzian correction. For the sample crystallized at $55^{\circ} \mathrm{C}$, the first $(\mathrm{P} 1)$ and the second (P2) peak positions are at $q_{1}=0.277 \mathrm{~nm}^{-1}$ and $q_{2}=0.481 \mathrm{~nm}^{-1}$, respectively, corresponding to 23 and $13 \mathrm{~nm}$. Odd enough, the ratio between $q_{2}$ and $q_{1}$ is very close to $\sqrt{ } 3{ }^{3}$ This would not support a lamellar structure but rather a hexagonal one. However, while the position P1 varies with temperature as discussed above, the position $\mathrm{P} 2$ of the second peak is about the same for the samples crystallized at 55 and $45{ }^{\circ} \mathrm{C}$. Thus for samples crystallized at different temperatures $q_{2} / q_{1}$ is not constant, which prevents us from a simple assignment. The nearly constant position of the second peak (P2) suggests that the corresponding structure may be independent of the crystalline structure leading for the first peak (P1). The obvious solution is that P2 is related to the microphase separation between PCL (crystalline) and PEO (amorphous). We shall see that several experiments involving crystallization of the PEO block support this hypothesis.

To induce crystallization and melting of PEO, step cooling and heating were applied to the sample crystallized at $45^{\circ} \mathrm{C}$. Figures 4(a), 4(b), 5(a), and 5(b) show the x-ray scat-
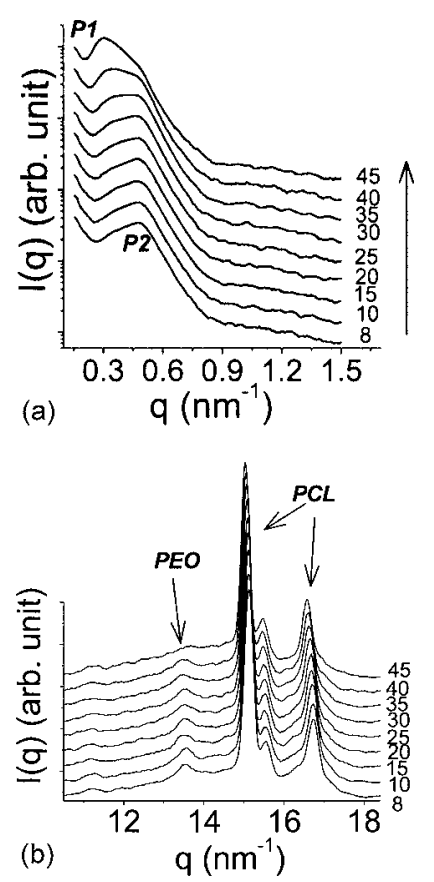

FIG. 5. (a) SAXS and (b) WAXS patterns during stepwise heating of PCL$b$-PEO initially crystallized at $45^{\circ} \mathrm{C}$ and stepwise cooled down to $8{ }^{\circ} \mathrm{C}$.

tering measurements during these processes. Initially, at $45^{\circ} \mathrm{C}$ only the PCL block crystallizes and PEO is in the amorphous state. During cooling, WAXS [Figs. 4(b) and 5(b)] reveals PEO crystallizing at about $15^{\circ} \mathrm{C}$. The heating process detects melting of the PEO crystals between 40 and $45^{\circ} \mathrm{C}$. The big difference $\left(>25^{\circ} \mathrm{C}\right)$ between the crystallization and melting temperatures tells us that nucleation of PEO blocks requires a large supercooling. This suggests isolated PEO domains in the microphase separated PEO- $b$-PCL system with crystallized PCL blocks, requiring homogeneous nucleation for subsequent PEO crystallization.

Crystallization of the PEO block during cooling [Fig. $\left.4(\mathrm{a}), 15^{\circ} \mathrm{C}\right]$ is accompanied by a sharp decrease of the intensity of the first SAXS peak (P1) and a slight shift of its position to larger $q$ values. This suggests a reduction of both the long spacing and the density contrast in the corresponding structure. On the other hand, the intensity of the second SAXS peak (P2) attributed to PEO increases without any obvious shift of its position. Melting of PEO crystals during heating [Fig. 5(a)] induces changes in the opposite direction as for crystallization. The different behaviors of these two peaks upon crystallization and melting of PEO block support the assignment that they have a rather independent structural origin.

Figure 6(a) shows the SAXS patterns from a sample initially crystallized at $58{ }^{\circ} \mathrm{C}$ before and after cooling down to the crystallization temperature of PEO. The high initial crystallization temperature for the PCL block leads to an improved separation of the two peaks after the crystallization of PEO even without a Lorentzian correction. As shown in the insertion of Fig. 6(a), the second peak (P2) is already present in the initial crystallized sample. Similar to the sample crystallized at $45{ }^{\circ} \mathrm{C}$, the same opposite trend of intensity and 

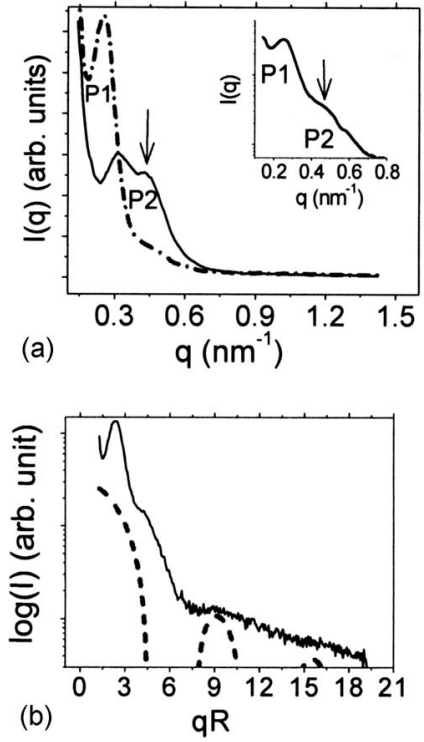

FIG. 6. (a) SAXS patterns of PCL- $b$-PEO crystallized at $58^{\circ} \mathrm{C}$ (dotted line) and subsequently cooled down to $22^{\circ} \mathrm{C}$ (solid line). Insert: pattern at $58{ }^{\circ} \mathrm{C}$ with a logarithmic intensity scale. (b) SAXS pattern of the sample crystallized at $58{ }^{\circ} \mathrm{C}$ (solid) together with a calculation of the form factor of spheres with a diameter of $9 \mathrm{~nm}$ (dot).

position of these two peaks was observed, which demonstrates that they indeed correspond to two independent structures.

As the first peak (P1) is directly correlated with the crystallization of the PCL block, which takes the major volume fraction in the system, a lamellar structure can be assigned to the first peak (P1). Though the crystallization of the PCL block induces the appearance of the second peak (P2), the increase of its intensity is further triggered by the crystallization of PEO, which increases the density contrast rather than the domain size. It is reasonable that PEO domains are responsible to the second peak (P2). A 17\% volume fraction of PEO should locate in the sphere region of the generic phase diagram of block copolymer. ${ }^{3}$ Figure 6(b) gives a simple calculation of the form factor of sphere with a diameter of $9 \mathrm{~nm}$. As the structure factor dominates at low $q$, the calculation is dedicated more to the high $q$. It fits reasonably well to the SAXS pattern from the sample crystallized at $58{ }^{\circ} \mathrm{C}$, especially the peak at $q R$ around 9 , which does not belong to a high order of the structure factor. Thus the morphology of the highly asymmetric PCL- $b$-PEO block copolymer is more likely to be a combination of lamellae and spheres.

Direct evidence of the overall morphology of the PCL$b$-PEO block copolymer was obtained from AFM. Figure 7 presents an AFM phase image of a sample initially crystallized at $45{ }^{\circ} \mathrm{C}$. A fresh surface was cut at liquid $\mathrm{N}_{2}$ temperature with PCL- $b$-PEO embedded in a polypropylene disk. The real space image of Fig. 7 shows a combination of lamellae and deformed spheres or a necklace kind of structure, confirming the interpretation of the SAXS results given above. As the morphology resulted from a nonthermodynamic equilibrium process, one would not expect a perfect structure as that from microphase separation. ${ }^{3}$

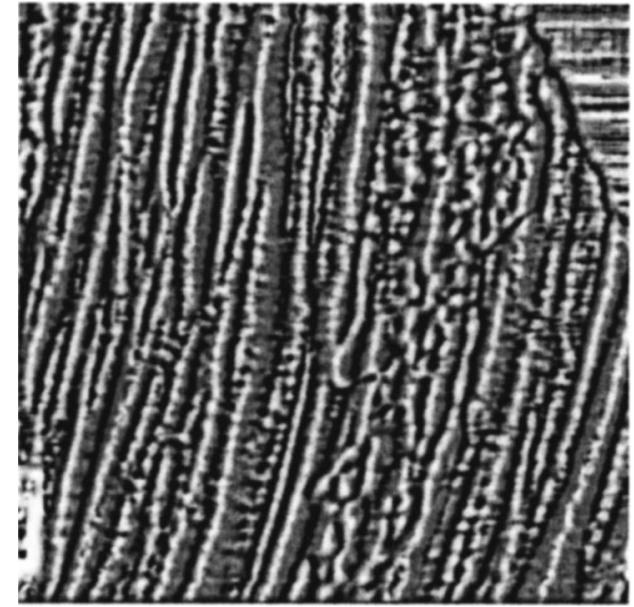

FIG. 7. AFM phase image $\left(1.5 \times 1.5 \mu \mathrm{m}^{2}\right)$ of PCL- $b$-PEO crystallized at $45^{\circ} \mathrm{C}$.

\section{DISCUSSION}

On the basis of the experimental results we can construct a full picture of the phase behavior of the PCL- $b$-PEO block copolymer that is schematically presented in Fig. 8. Note that Fig. 8 is only an ideal cartoon for the actual morphology, which is far from perfect as shown in Fig. 7. The starting point before the onset of any crystallization is a homogeneous melt [Fig. 8(a)] as supported by both SAXS and rheological measurements. Upon cooling the PCL blocks crystallize into a lamellar structure, which contributes to the first peak (P1) in the SAXS pattern (see Fig. 1). The long spacing is denoted as $d_{1}$ in Fig. 8(b). The $40 \%$ crystallinity of the PCL homopolymer is adopted in the cartoon. ${ }^{32}$ Accompanying the crystallization of the PCL block, microphase separation occurs between the PCL and PEO blocks. This is evidenced by the appearance of the second peak (P2) in SAXS patterns at high temperature where the PEO block is still in molten state (see Figs. 4-6). The corresponding period is denoted as $d_{2}$ in Fig. 8(b). As the microphase separation and the crystallization of the PCL block occur simultaneously, we anticipate a dynamic coupling and competition between these two phase transitions., ${ }^{2,11}$ However, the experiments [see Fig. 3(c)] indicate that the crystallization of PCL follows the typical pattern of homopolymers in spite of the presence of the PEO block. Nevertheless, the density fluctuation induced by the microphase separation can be expected to promote the nucleation of PCL crystals.

To discuss the intensity of the various peaks we denote the amorphous and crystalline densities of each block as given in Table I by $\rho_{a, \mathrm{PCL}}, \rho_{c, \mathrm{PCL}}, \rho_{a, \mathrm{PEO}}$, and $\rho_{c, \mathrm{PEO}}$. Note that

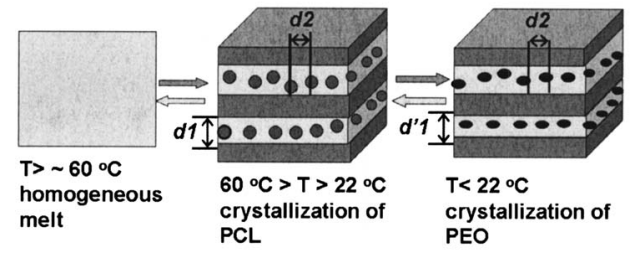

FIG. 8. Model illustrating the evolution with temperature of the morphology of the PCL- $b$-PEO block copolymer. 
here we use mass density to represent electron density due to the equivalence in terms of a relative comparison. The intensity of SAXS peak P1 $\left[d_{1}\right.$ in real space, Fig. 8(b)] is determined by the density difference between $\rho_{c, \mathrm{PCL}}$ and $\bar{\rho}$, some average of $\rho_{a, \mathrm{PCL}}$ and $\rho_{a, \mathrm{PEO}}$ as indicated in Fig. $8(\mathrm{~b})$. Compared to this value the difference $\rho_{a, \mathrm{PCL}}-\rho_{a, \mathrm{PEO}}$ is expected to be much smaller. The latter difference determines the intensity of the second SAXS peak (P2), which indeed is much lower than P1 (Figs. 3 and 6).

Further decreasing the temperature, the PEO spheres crystallize [Fig. 8(c)]. In terms of the intensity of SAXS peak $\mathrm{P} 1$, the contrast is now determined by the density difference between $\rho_{c, \text { PCL }}$ and $\bar{\rho}^{\prime}$, a new average of $\rho_{a, \mathrm{PCL}}$ and $\rho_{c, \mathrm{PEO}}$. Evidently $\bar{\rho}^{\prime}>\bar{\rho}$, leading to a decrease of the intensity. On the other hand, regarding SAXS peak P2, the density difference $\rho_{a, \mathrm{PCL}}-\rho_{a, \mathrm{PEO}}$ is now replaced by the larger $\rho_{a, \mathrm{PCL}}-\rho_{c, \mathrm{PEO}}$. Thus, the crystallization of PEO increases the intensity of the second peak $\mathrm{P} 2$. As a result, the intensities of these two peaks follow an opposite trend when the PEO spheres crystallize.

Let us now discuss the influence of the crystallization of PEO on the positions $\left(q_{1}\right.$ and $\left.q_{2}\right)$ of the two SAXS peaks. Peak P1 shifts significantly to higher $q$ values, while no obvious position change of the second peak P2 is observed. This result is not obvious from the model of Fig. 8 and suggests that the crystallization of PEO adopts a preferred orientation. The PEO spheres are confined in the hard crystallized PCL layers in vertical direction [see Fig. 8(b)] and soft amorphous PCL medium lateral sides. The crystallization of PEO may sense this difference and shrink more in the direction of hard confinement rather than at the soft sides. This would bring $q_{1}$ up and keep $q_{2}$ unchanged.

Our observations on the highly asymmetric PCL- $b$-PEO block copolymer may be of interest for the crystallization of homopolymers. As is well known, high-molecular mass polymers such as PE and PP have many chain defects such as stereo defects and comonomers. Taking these defects into account, HDPE and iPP naturally fall in the group of "(highly) asymmetric copolymers." Note that for many polymers the difference of tacticity is sufficient to induce phase separation in the melt. ${ }^{38-41}$ Though this has not been observed for the melt of common iPP or HDPE, upon cooling down to the supercooled melt region coupling between crystallization and microphase separation might occur. ${ }^{42,43}$ It has been well noted that the modulated lamellae reported earlier for an asymmetric PEO- $b$-PS block copolymer ${ }^{10}$ are rather similar to the granular morphology of sPP and other (co-)polymers described by Strobl $^{44}$ and others. Such a similarity of morphology implies that a similar underlying mechanism is at work. We speculate that the granular morphology may not be induced by crystallization but rather by the coupling and competition between crystallization and microphase separation. Microphase separation is a weak firstorder phase transition, generally thought to be easily overruled by crystallization and ignored in the case of homopolymer crystallization. However, the phase behavior of the present PCL- $b$-PEO system and our previous work on asymmetric PEO- $b$-PS block copolymers demonstrates that such a weak phase transition could induce density fluctuation and thus enhance nucleation. ${ }^{45}$ The kinetic competition of the two phase transitions leads to the formation of nonstandard morphologies, such as the granular structure or perforated lamellae.

Taking a possible microphase separation in common homopolymers into account, it might be possible to explain several experimental observations that could not be elucidated so far by polymer crystallization alone. For example, the unexplained two uncorrelated SAXS peaks from several homopolymers, such as polyoxymethylene and $\mathrm{PE},{ }^{46}$ could be interpreted on this basis. Further experiments are in progress to explore these possibilities.

\section{CONCLUSIONS}

Using in situ simultaneously small- and wide-angle $\mathrm{x}$-ray scattering, the morphology of a highly asymmetric double crystallizable PCL- $b$-PEO block copolymer has been studied. With molecular masses $M_{n}$ of 24000 and 5800 for PCL and PEO blocks, respectively, no microphase separation occurs in the melt. Decreasing the temperature to the supercooled melt, coupling and competition between the microphase separation and the crystallization occurs, which results in a structure of crystallized lamellar stacks of PCL with amorphous PEO spheres embedded in the noncrystalline regions. The amorphous $\mathrm{PEO}$ spheres crystallize at a lower temperature with a preferred orientation due to the confinement of hard PCL crystalline lamellae in one direction only. The morphological similarity between highly asymmetric block copolymers and some homopolymers suggests coupling and competition between crystallization and microphase separation as a similar underlying mechanism.

\section{ACKNOWLEDGMENTS}

The authors want to thank Ernst Prins (Amsterdam) for his invaluable technical help with the experiments. This work is supported by the National Science Foundation (50503015) as well as the "One Hundred Talent Scientist" program of the Chinese Academy of Sciences.

${ }^{1}$ M. Muthukumar, C. K. Ober, and E. L. Thomas, Science 277, 1225 (1997).

${ }^{2}$ E. A. Di Marzio, Prog. Polym. Sci. 24, 329 (1999)

${ }^{3}$ I. W. Hamley, The Physics of Block Copolymers (Oxford University Press, New York, 2000).

${ }^{4}$ Y. L. Loo, R. A. Register, and A. J. Ryan, Phys. Rev. Lett. 84, 4120 (2000).

${ }^{5}$ G. Reiter, G. Castelein, J. U. Sommer, A. Röttele, and T. Thurn-Albrecht, Phys. Rev. Lett. 87, 226101 (2001).

${ }^{6}$ L. Zhu, B. H. Calhoun, Q. Ge, R. P. Quirk, and S. Z. D. Cheng, Macromolecules 34, 6649 (2001); Polymer 42, 5829 (2001); J. Am. Chem. Soc. 122, 5957 (2000).

${ }^{7}$ S. Nojima, N. Kikuchi, A. Rohadi, S. Tanimoto, and S. Sasaki, Macromolecules 32, 3727 (1999).

${ }^{8}$ J. Fu, B. Luan, C. Y. Pan, B. Y. Li, and Y. C. Han, Macromolecules 38, 5118 (2005)

${ }^{9}$ W. H. de Jeu, in Polymer Crystallization: Observations, Concepts and Interpretations, Springer Lecture Notes in Physics Vol. 606, edited by G. Reiter and J.-U. Sommer (Springer, Berlin, 2003).

${ }^{10}$ L. B. Li, D. Lambreva, and W. H. de Jeu, J. Macromol. Sci., Phys. 43, 59 (2004); L. B. Li, Y. Serero, M. H. J. Koch, and W. H. de Jeu, Macromolecules 36, 529 (2003).

${ }^{11}$ A. J. Muller, V. Balsamo, and M. L. Arnal, Adv. Polym. Sci. 190, 1 (2005). 
${ }^{12}$ J. Albuerne, L. Marquez, A. J. Muller, J. M. Raquez, Ph. Dege'e, Ph. Dubois, V. Castelletto, and I. W. Hamley, Macromolecules 36, 1633 (2003).

${ }^{13}$ Y. Chen and G. L. Baker, J. Am. Chem. Soc. 121, 6962 (1999).

${ }^{14}$ G. Maglio, A. Migliozzi, and R. Palumbo, Polymer 44, 369 (2003).

${ }^{15}$ M. L. Arnal, V. Balsamo, F. López-Carrasquero, J. Contreras, M. Carrillo, H. Schmalz, W. Abetz, E. Laredo, and A. J. Muller, Macromolecules 34, 7973 (2001).

${ }^{16}$ B. Bogdanov, A. Vidts, E. Schacht, and H. Berghmans, Macromolecules 32, 726 (1999).

${ }^{17}$ D. Shin, K. Shin, K. A. Aamer, G. N. Tew, T. P. Russell, J. H. Lee, and J. Y. Jho, Macromolecules 38, 104 (2005).

${ }^{18}$ J. R. Sun, X. S. Chen, C. L. He, and X. B. Jing, Macromolecules 39, 3717 (2006)

${ }^{19}$ L. Sun, Y. X. Liu, L. Zhu, B. S. Hsiao, and C. A. Avila-Orta, Polymer 45, 8181 (2004).

${ }^{20}$ S. Nojima, Y. Akutsu, A. Washino, and S. Tanimoto, Polymer 45, 7317 (2004).

${ }^{21}$ C. L. He, J. R. Sun, C. Deng, T. Zhao, M. X. Deng, X. S. Chen, and X. B. Jing, Biomacromolecules 5, 2042 (2004).

${ }^{22}$ C. L. He, J. R. Sun, T. Zhao, Z. K. Hong, X. L. Zhuang, X. S. Chen, and X. B. Jing, Biomacromolecules 7, 252 (2006).

${ }^{23}$ I. W. Hamley, P. Parras, V. Castelletto, R. V. Castillo, A. J. Muller, E. Pollet, P. Dubois, and C. M. Martin, Macromol. Chem. Phys. 207, 941 (2006).

${ }^{24}$ I. W. Hamley, V. Castelletto, R. V. Castillo, A. J. Muller, C. M. Martin, E. Pollet, and Ph. Dubois, Macromolecules 38, 463 (2005).

${ }^{25}$ J. M. Harris, in Poly(Ethylene Glycol) Chemistry: Biotechnical and Biomedical Applications, edited by J. M. Harris (Plenum, New York, 1992), p. 7.

${ }^{26}$ C. G. Pitt, In Biodegradable Polymers as Drug Delivery Systems, edited by M. Chasin and R. Langer (Dekker, New York, 1990), p. 71.

${ }^{27}$ F. H. Meng, C. Hiemstra, G. H. M. Engbers, and J. Feijen, Macromolecules 36, 3004 (2003)

${ }^{28}$ R. Perret and A. Skoulios, Makromol. Chem. 156, 143 (1972).
${ }^{29}$ P. Cerrai, M. Tricoli, and F. Andruzzi, Polymer 30, 338 (1989).

${ }^{30}$ S. Nojima, M. Ono, and T. Ashida, Polym. J. (Tokyo, Jpn.) 24, 1271 (1992).

${ }^{31}$ Z. Gan, B. Jiang, and J. Zhang, J. Appl. Polym. Sci. 59, 961 (1996).

${ }^{32}$ G. Strobl, Prog. Polym. Sci. 31, 398 (2006).

${ }^{33}$ B. Wunderlich, Nucleation and Growth, Macromolecular Physics Vol. 2 (Academic, New York, 1973).

${ }^{34}$ E. A. DiMarzio, C. M. Guttman, and J. D. Hoffmann, Macromolecules 13, 1194 (1980).

${ }^{35}$ M. D. Whitmore and J. Noolandi, Macromolecules 21, 1482 (1989).

${ }^{36}$ W. Y. Chen, J. X. Zheng, S. Z. D. Cheng et al., Phys. Rev. Lett. 93, 028301 (2004).

${ }^{37}$ L. B. Li, Z. Y. Zhong, W. H. de Jeu, P. J. Dijkstra, and J. Feijen, Macromolecules 37, 8641 (2004).

${ }^{38}$ D. Lohse, Polym. Eng. Sci. 26, 1500 (1986).

${ }^{39}$ R. Thomann, J. Kressler, B. Rudolf, and R. Mulhaupt, Polymer 37, 3635 (1996).

${ }^{40}$ P. Choi and W. L. Mattice, J. Chem. Phys. 121, 8647 (2004).

${ }^{41}$ C. Wang, C. C. Lin, and L. C. Tseng, Polymer 47, 390 (2006).

${ }^{42}$ W. B. Hu, V. B. F. Mathot, and D. Frenkel, Macromolecules 36, 2165 (2003).

${ }^{43}$ W. B. Hu and V. B. F. Mathot, Macromolecules 37, 673 (2004).

${ }^{44}$ G. Strobl, Eur. Phys. J. E 3, 165 (2000).

${ }^{45}$ P. R. ten Wolde and D. Frenkel, Science 277, 1975 (1997).

${ }^{46}$ P. H. Geil, Polymer 41, 8983 (2000).

${ }^{47}$ V. Crescenzi, G. Manzini, G. Galzolari, and C. Borri, Eur. Polym. J. 8, 449 (1972).

${ }^{48}$ Y. Chatani, Y. Okita, and H. Tadokoro, Polym. J. (Tokyo, Jpn.) 1, 555 (1970).

${ }^{49}$ L. Zhu, S. Z. D. Cheng, B. H. Calhoun, Q. Ge, R. P. Quirk, E. L. Thomas, B. S. Hsiao, F. Yeh, and B. Lotz, Polymer 42, 5829 (2001).

${ }^{50}$ H. L. Chen, L. J. Li, W. C. Ou-Yang, J. C. Hwang, and W. Y. Wong, Macromolecules 30, 1718 (1997).

${ }^{51}$ G. C. Alfonso and T. P. Russell, Macromolecules 19, 1143 (1986). 